\title{
Artificial intelligence (AI)-An Inflection Point In the Global History and its Significance For a National Strategy
}

\author{
Dinesh Kadam ${ }^{1}$

\begin{abstract}
${ }^{1}$ Department of Plastic and Reconstructive Surgery, A I Institute of Medical Sciences and A J Hospital and Research Centre, Mangalore, Karnataka, India
\end{abstract}

Indian J Plast Surg 2019;52:145-147

"AI is the new electricity"-Andrew Ng.

Andrew Ng, ${ }^{1}$ the Director of the Stanford Artificial Intelligence Laboratory, equates the age of Artificial Intelligence (AI) to the discovery of electricity for allowing inexhaustible applications into all walks of life. AI might be the single largest technology revolution of our times, and we are now living in an AI-led era already. Be it, Amazon Alexa, Apple Siri, or Google Assistant, our smartphones now are equipped with a personal assistant powered by AI with nearly $90 \%$ accuracy and dependability. From live "chatbots," to smart suggestions for travel, shopping, or restaurants, AI has invariably impacted our everyday lives. The buzz around this incredible technology is beguiling and poised for a paradigm shift in our lives.

\section{Can a Machine Be Made to Think?}

That question unanswered in the past has now been answered in the affirmative albeit in a limited set of circumstances. Machines can now be trained to do tasks of various complexities with a defined output of measure, known as "machine learning" (ML). This ML can handle a small quantity of data and provide a defined outcome. Going to the next level is "deep learning" (DL), which is similar to the network of neurons of the brain, with stacked layers and connecting in a random pattern. This deep neural network can handle large quantities of data, learns by itself, and makes intelligent decisions and performs complex tasks; in other words, it mimics the functions of the brain. Both machine learning and deep learning are a subset of AI. It simply means the ability of machines to perform cognitive tasks such as thinking, perception, learning, language skills, problem solving, and decision making akin to natural human intelligence. Based on their capacities, they are also called "weak AI" and

Address for correspondence Dinesh Kadam, MS, DNB, MCh, Department of Plastic and Reconstructive Surgery, A J Institute of Medical Sciences and A J Hospital and Research Centre,Mangalore 575006, Karnataka, India (e-mail: drkadam@yahoo.co.in). "strong AI," which are essentially machine learning and deep learning, respectively. ${ }^{2}$

The concept of AI is not new, and researcher Alan Turing, the great British code breaker in the second world war had done considerable work in this field but it was John McCarthy who coined the term AI in $1955 .^{3}$ This field however remained very limited in its scope because the data was very small. Over a period of time the data has increased exponentially to the current estimate of 4.4' Zetabytes' (ZB) and is likely to rise to $44 \mathrm{ZB}$ or 44 trillion Gigabytes (GB) by the year 2020. This is called "big data" and data mining is "data science." The cost of hard drive per GB has fallen drastically from $\$ 50,000 / G B$ in 1980 to 2 cents/GB today. AI is already in use in many industries and web applications. It is expected that $70 \%$ of enterprises will implement AI by the end of 2019.

AI technology is at an emerging and evolving juncture. India, a growing economy and the second-most populous nation, has a tremendous opportunity to carve its needspecific and robust AI ecosystem. With the inauguration of India's first dedicated AI research institute ${ }^{4}$ in Mumbai, and the NITI Aayog the national think tank bringing out a discussion paper on $\mathrm{AI}$ in the year 2018 , the ground work has been laid down in order to gain an early momentum for India in the global development of AI. ${ }^{5}$ Taking this forward, the government of India has announced plans to establish a national centre for AI and a national program for AI.This year, the NITI Aayog has drawn up a plan providing Rs. 7,500 crores for creating an institutional framework for $\mathrm{AI}$ in the country. ${ }^{6}$ This has been a much needed impetus for India to catch up with her international peers.

Globally, during the last two years, countries like China, the Unites States, Japan, the United Kingdom, and France have announced their national policy positions on AI. It is estimated that by $2030,26 \%$ of the GDP of China and $10 \%$ of the GDP of the United Kingdom will be sourced from
(C)2019 Association of Plastic Surgeons of India

\section{License terms}

10.1055/s-0039-1700367

ISSN 0970-0358. 
AI-related businesses. ${ }^{5}$ China is leading the race and has strategized to become a global leader by 2030 . With a robust thrust from the Chinese government to invest and incorporate diverse $\mathrm{AI}$ applications such as face recognition surveillance and AI chip technology, they have got an early start to lead the way in harnessing A.I. Currently, China harbours the biggest cluster of AI scientists and has a well-structured program in place. In this technological race with high geopolitical stakes, both China and the United States are head and shoulder above the rest. ${ }^{7}$ This dominance over the technology is cornered by a handful of tech giants, which includes Google, Apple, Amazon, Facebook, and Microsoft (GAFAM) of the United States and Baidu, Alibaba, Tencent, and Xiaomi (BATX) of China. These leviathans together have a market capitalization of $\$ 4.5$ trillion ( $\$ 3,438$ billion for GAFAM and $\$ 1,132$ billion for BATX) in the AI technology expansion. ${ }^{8}$ Despite global attention and expectation, the benefits of this revolutionary technology will be limited to less than $50 \%$ of humanity unless a wide range of societal domains is included.

\section{An Inflection Point in Indian Strategy}

In what manner and for what use is a' labour surplus' developing economy like India is looking at AI?

AI can be used both for harnessing information and knowledge and in turn improving expertise in various fields. The NITI Aayog in its vision paper has focused on reforms in five important sectors or domains. (1) healthcare to improve access, affordability, and quality; (2) agriculture to enhanced farmers' income, farm productivity, and reduction of wastage (3) education to improve access and quality; (4) smart cities and infrastructure for the urban population; and (5) smart mobility and transportation to ease traffic congestion and improve safety.

Setup on a "public-private partnership" model, the Wadhwani Institute for $\mathrm{AI}^{4}$ has already worked on three core issues-maternal, infant and child health, in detecting low birthweight babies with the virtual weighing machine, on multiple challenges in tuberculosis control, and lastly in cotton farming to reduce crop losses which is the third largest agricultural yield in India mostly of small farmers. There are already more than $1,500 \mathrm{AI}$ startups in India, to cater to local and global needs. ${ }^{9}$

The nations of the developing part of the world share common challenges in sectors such as health, education, infrastructure, agriculture, and urbanization. With IT capability, India has the potential to becoming a "global player" to produce scalable solutions for similar problems. In effect, the solutions developed and applied to India can benefit $40 \%$ of the rest of the world, and this is in fact a tremendous opportunity for the country to assume its position as a trailblazer for the emerging economies. The government is rightly exploring the potential to provide a destination for an "AI garage." The NITI Aayog estimates adding $\$ 1$ trillion to the Indian economy by way of new AI solutions in the next 15 years which will boost India's annual growth by 1.3 percentage points by $2035 .{ }^{5}$

\section{$\mathrm{Al}$ in Health Care}

With data revolution and effective data mining, the application of AI has brought the health care sector vastly on the way of the transformation. This impact is seen across the specialties, including radiology, dermatology, neurology, ophthalmology, oncology, cardiology, genetics, emergency medicine, and so on during the past few years. The medical image recognition technique by deep learning has produced predictable accuracy in interpretation, which are at par with those of trained radiologists. Despite the perceived threat of radiologists losing jobs, the near future likely to witness the increased collaboration between radiologists and the technology to significantly enhance clinical benefits in terms of accuracy, cost, speed, and also their reach to remote areas. Similarly, Google AI has developed "computer vision" technique to detect diabetic retinopathy and in the diagnosis of malignancy utilizing digital pathology. ${ }^{10}$ Robotics powered by $\mathrm{AI}$ has been in use for demanding and high-precision surgeries.

The AI in healthcare can help to address issues such as poor connectivity and limited supply of healthcare professionals in rural areas which at present constitute significant barriers. This can be achieved through the implementation of cases such as AI-driven image diagnostics, clinical decision support, personalized treatment, predictive population risk stratification, early identification of potential pandemics, and tools for patients to manage their own illnesses.

\section{Al in Plastic Surgery}

Currently, the impact on the field of plastic surgery is limited to specific tasks such as quantification of burn wound size, monitoring of vascular perfusion following microvascular surgery, and to facilitate accurate diagnosis of craniofacial anomalies based on computed tomography (CT) images (with $98 \%$ specificity and $92 \%$ sensitivity). ${ }^{11,12}$ It has been found useful in orthognathic surgery due to improved diagnostics, therapeutic planning, computer-assisted appliances, intraoperative navigations, and follow up of patients. ${ }^{13}$ The potential for use in aesthetic surgery, the results of which are largely subjective is also being explored with predictive tools for patient-perceived beauty. Intraoperative assessment of symmetry and precise anatomical position can be made out using an optical "head-mounted" display, and a higher degree of objectivity can be achieved. ${ }^{11} \mathrm{AI}$ has powered biomechanical neuroprosthesis to help in precise finger and wrist movements to provide a better grip.

AI technology has loomed over the horizon and it is here to stay. At this inflection point, it is a remarkable opportunity for all of us to be aware, and adapt to this change to supplement our technical capacity as well as selfempowerment. Lastly, no nation can now neglect this tool if it wants to keep pace with others and if it does so, it will be at its own peril.

\section{Conflict of Interest}

None. 


\section{References}

1 Wikipedia. Andrew Ng. https://en.wikipedia.org/wiki/Andrew_ Ng. Accessed July 10, 2019

2 TechTarget. Special report: artificial intelligence apps come of age. https://searchenterpriseai.techtarget.com/definition/ AI-Artificial-Intelligence. Accessed July 3, 2019

3 Artificial intelligence, the history and future-with Chris Bishop [video online]. https://youtu.be/8FHBh_OmdsM. Accessed July 16, 2019

4 Wadhwani AI website. https://www.wadhwaniai.org/. Accessed July 8, 2019

5 NITI Aayog. National strategy on artificial intelligence.https:// www.niti.gov.in/national-strategy-artificial-intelligence. Accessed August 12, 2019

6 Yogima Sharma. Niti Aayog proposes Rs 7,500-crore plan for artificial intelligence push. https://economictimes. indiatimes.com/news/economy/policy/niti-aayog-proposesrs-7500-crore-plan-for-artificial-intelligence-push/articleshow/69403255.cms?from=mdr. Accessed July 16, 2019

7 Neha Dewan. In the race for AI supremacy, has India missed the bus? https://economictimes.indiatimes.com/small-biz/ startups/features/in-the-race-for-ai-supremacy-has-india-missed-the-bus/articleshow/69836362.cms?from=mdr. Accessed July 29, 2019
8 Leaders League. GAFA vs BATX: to rule them all. https://www. leadersleague.com/en/news/gafa-vs-batx-to-rule-them-all. Accessed August 2, 2019

9 Shubham Singh. 10 artificial intelligence (AI) startups in India you should know. https://www.analyticsvidhya.com/ blog/2019/07/10-ai-startups-india/. Published July 2, 2019. Accessed Aug 5, 2019

10 Google AI website. https://ai.google/healthcare. Accessed August 16, 2019

11 Kim YJ, Kelley BP, Nasser JS, Chung KC. Implementing precision medicine and artificial intelligence in plastic surgery: concepts and future prospects. Plast Reconstr Surg Glob Open 2019;7(3):e2113

12 Kanevsky J, Corban J, Gaster R, Kanevsky A, Lin S, Gilardino M. Big data and machine learning in plastic surgery: a new frontier in surgical innovation. Plast Reconstr Surg 2016;137(5):890e-897e

13 Bouletreau P, Makaremi M, Ibrahim B, Louvrier A, Sigaux N. Artificial intelligence: applications in orthognathic surgery. J Stomatol Oral Maxillofac Surg 2019;120(4):347-354 